\title{
COLLAPSE SAFETY MARGIN-BASED DESIGN OPTIMIZATION OF STEEL STRUCTURES WITH CONCENTRICALLY BRACED FRAMES
}

\author{
Jeriniaina Sitraka Tantely ${ }^{1}$ and Zheng $\mathrm{He}^{1,2, *}$ \\ ${ }^{1}$ Department of Civil Engineering, Dalian University of Technology, Dalian, Liaoning, China \\ ${ }^{2}$ State Key Laboratory of Coastal and Offshore Engineering, Dalian University of Technology, Dalian, Liaoning, China \\ *(Corresponding author: E-mail: hezheng@dlut.edu.cn)
}

\section{A B S T RA C T}

Finding an optimum design based on collapse safety assessment for bracing systems in steel structures can result in a safe and economical design, and therefore is highly desirable. Several works in the literature have successfully applied various design methodologies for braced frames. The occurrence of unforeseen events outside the scope of these designs might jeopardize their structural integrity. Therefore, tools such as incremental dynamic analysis and modal pushover analysis have been developed to assess the probability of structural collapse. However, their implementation in the design process is challenging because their procedures are onerous and time-consuming. To overcome this issue, a straightforward method utilizing empirical equation to estimate the collapse margin of the structure is used. The proposed methodology uses the brace locations and sections as variables. A probabilistic analysis using multi-element removal identifies the bracing layouts, and explicit equations determine their optimal discrete sections. The methodology creates all the possible schemes, then identifies the optimal one that has the highest safety index based on a targeted collapse margin ratio. Through solving four typical examples of steel framed structures, the practicality, and accuracy of the proposed approach are proved.

\section{A R T I C LE H I S T O RY}

$\begin{array}{ll}\text { Received: } & 27 \text { August } 2017 \\ \text { Revised: } & \text { 27 July } 2018 \\ \text { Accepted: } & \text { 01 August } 2018\end{array}$

\section{K E Y W O R D S}

Steel structures;

Brace frames;

Collapse safety;

Probabilistic analysis;

Multi-element removal;

Safety index

Copyright $\odot 2019$ by The Hong Kong Institute of Steel Construction. All rights reserved.

\section{Notation}

The following symbols are used in this paper:

$A_{B}^{\text {opt }} \quad=$ optimal brace section;

$A_{b}^{\text {opt }} \quad=$ optimal discrete brace section;

$A_{B}^{\mathrm{s}} \quad=$ total area of the bracing system;

$A_{b}^{\mathrm{s}} \quad=$ database brace section;

$A_{y} \quad=$ pseudo-acceleration $(\mathrm{g})$;

$B \quad=$ binary matrix representing the bays;

$B^{\prime} \quad=$ modified binary matrix representing the bays;

$b_{T C} \quad=$ number of brace forming the type of configuration;

$C d t \quad=$ matrix candidate;

$c \_s p=$ count of the sub-parent;

$E \quad=$ elasticity modulus of the brace $(\mathrm{Mpa})$;

$f_{r} \quad=$ residual strength $(\mathrm{Mpa})$;

$f_{y} \quad=$ yield strength $(\mathrm{Mpa}) ;$

$h \quad=$ structural height with respect to the ground level $(\mathrm{mm})$

IM = intensity measure;

$K^{\text {opt }} \quad=$ optimal lateral shear stiffness;

$k_{c} \quad=$ negative slope stiffness;

$k_{e} \quad=$ initial stiffness;

$k_{s} \quad=$ second slope stiffness;

$L \quad=$ total length of the brace $(\mathrm{mm})$

$l \quad=$ effective length of the brace $(\mathrm{mm})$;

$N^{\mathrm{db}} \quad=$ number of designable bay;

$N^{\text {opt }} \quad=$ optimal number of the brace;

$N^{\text {tb }} \quad=$ number total of bay;

$N^{\mathrm{ub}} \quad=$ number of undesignable bay;

$n \quad=$ number of story;

Pos = total number of possible brace layouts of the structure;

$p c \quad=$ number of possible combination for the repartition of the braces;

pst = matrix containing all possible combinations for brace location;

$R_{c} \quad=$ collapse strength ratio;

$S_{D S} \quad=$ design spectral response acceleration parameter at short periods;

$S_{D 1} \quad=$ design spectral response acceleration parameter at a period of $1 \mathrm{~s}$;

$S I \quad=$ safety index;

$S P \quad=$ sub-parent;

$T_{L} \quad=$ long-period transition period;

$T_{S} \quad=S_{D 1} / S_{D S} ;$

$T_{-} s p=$ total number of sub-parent;

$T_{-} p=$ total number of parent;

$T_{0} \quad=0.2 S_{D 1} / S_{D S}$

$T_{1} \quad=$ period of the structure at $1 \mathrm{~s}$;

$u_{c} \quad=$ maximum displacement $(\mathrm{mm})$;

$u_{y} \quad=$ yield displacement $(\mathrm{mm})$;
$V \quad=$ story shear $(\mathrm{Mpa}) ;$

$X \quad=$ element of the matrix candidate;

$\alpha \quad=$ inclination angle of the brace (rad);

$\alpha_{c} \quad=k_{c} / k_{c}$

$\alpha_{s} \quad=k_{s} / k_{e}$

$\beta \quad=$ dispersion of the $I M$

$\Delta \delta_{\mathrm{f}} \quad=$ flexure drift $(\mathrm{mm})$

$\Delta \delta_{\mathrm{s}}^{\text {opt }} \quad=$ optimal shear drift $(\mathrm{mm})$

$\Delta \delta^{\text {target }}=$ target drift $(\mathrm{mm})$;

$\delta^{\text {opt }} \quad=$ optimal lateral deflection $(\mathrm{mm})$;

$\delta_{\mathrm{s}}^{\text {opt }} \quad=$ optimal shear displacement $(\mathrm{mm})$;

$\delta_{\mathrm{f}} \quad=$ flexure displacement $(\mathrm{mm})$

$\zeta \quad=$ damping $(\%)$;

$\theta \quad=$ median of the fragility function;

$\mu \quad=$ index ratio of the overturning moment distribution;

$\mu_{c} \quad=$ ductility coefficient; and

$\rho^{\text {total }}=$ total rotation of the slab $(\mathrm{rad})$.

\section{Introduction}

The basic design of braced steel structures focuses on their structural resistance under frequent and rare earthquakes. With the evolution of the computer-aided design, a wide range of design objectives integrates the bracing final layout, including topological, geometrical and economic aspects [1]. The occurrence of severe events outside the scope of the designs above might compromise the integrity of the braced steel structures. The urge to understand the unforeseen events leads the researchers to develop innovative ways to assess the structural collapse. However, their implementation in the structural design remains challenging because the procedures used for estimating the structural safety are often onerous and time-consuming. A design methodology based on safety criteria would provide a promising tool to obtain an optimal braced steel structures design.

Structural collapse assessment extended to a wide range of engineering applications in the past two decades. The breakthrough in the understanding of the structural collapse due to seismic events was the introduction of the incremental dynamic analysis (IDA) by Vamvatsikos and Cornell [2]. The outcome of IDA permits to create a collapse probability curve for the studied structure. Although the researchers are unanimous concerning the efficiency and reliability of the IDA, they think that it is computationally demanding and remains a time-consuming process. They have solved these restrictions by approximating the fragility curve with a few series of time history analysis (THA) [3-5]. Liu et al. [6] improved the efficiency of the dynamic THA by considering geometric nonlinearity. The pushover analysis is also an acceptable method for seismic design [7, 8]. On the other hand, Han et al. [9] and Moon et al. [10] completely avoided the use of THA by establishing empirical equations. 
Although this method has some limitations, it gives an acceptable approximation of the fragility curve and tremendously improves the calculation time of the collapse assessment. FEMA [11] extended the use of the fragility curve by proposing a collapse margin ratio $(C M R)$, which becomes the primary parameter associated with the evaluation of structural safety. The higher the $C M R$ value is, the safer the structure will be. However, several variables related to a given ground motion $[12,13]$, and to the structure $[14,15]$ might increase or decrease the $C M R$. Also, Xian, et al. [14] stated that $C M R$ is firmly a casedependent factor. Therefore, the utilization of some optimization algorithm that evolves the use of source models $[16,17]$ to get the safest structure conflict with the present understanding of the $C M R$.

Thus, the utilization of $C M R$ as design criteria of the bracing system for a given steel structures implies the identification of several brace schemes, i.e. brace locations and optimal discrete sections. The issue of the brace locations involves the use of probability, more precisely, the occurrence of a given brace combination at a given floor and the interaction between that brace combination to the others from different floor level. The closest example of this procedure is the member removal method [18], where, in this work, the focus of the removal is the braces. Gholizadeh and Poorhoseini [19] use predefined brace combinations as variable to search the optimal layout of the steel braced frame. However, in this work, the authors consider every possible layout to obtain their respective optimal total brace sections, which increases the complexity of their computational work. Therefore, the proposed design algorithm should calculate a single optimal section for all the braces at a given floor, and utilize a database composed of the available commercial brace section as variables for the discrete results [20]. This approach may take more time and effort, but higher accuracy could be achieved.

The scope of this work is to propose a design optimization of steel structures using concentric braces based on collapse safety assessment. The brace locations and sections are the variables of this investigation. This paper is organized as follows. The next section presents the methodology to identify all possible bracing locations; followed by the elaboration of the brace sizing optimization, and finalized by the solution algorithm based on $C M R$ calculation to get the optimal design. Examples are presented to show the application and efficacy of the proposed method.

\section{Possible brace schemes using probabilitic analysis of multi-element removal}

\subsection{Designable matrix}

The idea of designable matrix comes from the fact that in actual constructions, engineers do not always have the free choice to install the braces in any locations of the building, due to architectural constraints or owners' preferences. The bay where the installation of braces is not permitted is named undesignable bay. To quickly count the number of the designable and undesignable bays in a given structure, an initial matrix $B$ ( $B$ stands for bays), representing an elevation view of the building, is established. The elements of this matrix form a binary representation, as follow,

$B^{n \times N^{\natural}}=\left\{\begin{array}{cc}B_{i, j}=0 & \text { Undesignable bay } \\ B_{i, j}=1 & \text { Designable bay }\end{array} \quad\left(i=1, \ldots, n ; j=1, \ldots, N^{\mathrm{tb}}\right)\right.$

where $n$ and $N^{\mathrm{tb}}$ are the number of story and the total number of bay, respectively; the subscript $i$ and $j$ represents the story and the bay. Eq. 2 gives the expression of $N^{\mathrm{tb}}$.

$N_{i}^{\mathrm{tb}}=N_{i}^{\mathrm{db}}+N_{i}^{\mathrm{ub}}$

with

$N_{i}^{\mathrm{db}}=\sum_{j=1}^{N^{\mathrm{b}}} B_{i, j}$

where $N_{i}^{\mathrm{db}}$ and $N_{i}^{\mathrm{ub}}$ represent the number of the designable and undesignable bays at the $i$-th story, respectively.

\subsection{Matrix brace locations}

A probabilistic analysis of multi-element removal identifies all possible designable matrix, also known as parents $(P)$, regarding the matrix $B$. First, the algorithm counts and locates each designable bay $(D B)$, by creating a vector $D B$ with Eq. 4.

$D B_{k}^{i}=j \quad$ for $B_{i, j}=1 \quad\left(i=1, \ldots, n ; j=1, \ldots, N_{i}^{\mathrm{tb}}, k=1, \ldots, N_{i}^{\mathrm{db}}\right)$

Then, to get all possible combinations for the brace location at the $i$-th story, the authors use the command "nchoosek" in Matlab as shown below.

pst $=$ nchoosek $\left(D B_{k}^{i}, u\right)$

where pst is a matrix containing all possible combinations of the elements of $D B_{k}{ }^{i}$ taken $u$ at a time, and $u$ is a number increasing from 0 to $\left(N_{i}^{\mathrm{db}}-1\right)$. The matrix pst has u columns and $q ! /((q-u) ! u !)$ rows, where $q$ is the length of $D B_{k}{ }^{i}$.

The first sub-parent, $S P$, for the $i$-th story is $B_{i}$ itself, and then the remaining ones utilize the matrix pst as a reference by replacing the element " 1 " in $B_{i}$ by " 0 " corresponding to the brace location in each row of pst.

$S P_{i, c_{-} s p}=\left\{\begin{array}{c}B_{i} \text { if } u=0 \\ B_{i}^{\prime} \text { if } u=1, \ldots, N_{i}^{\mathrm{db}}-1\end{array}\right.$

with

$B_{i, j}^{\prime}= \begin{cases}0 & \text { if } p s t_{i}=j \& B_{i, j}=0 \\ 0 & \text { if } p s t_{i}=j \& B_{i, j}=1 \\ 0 & \text { if } p s t_{i} \neq j \& B_{i, j}=0 \\ 1 & \text { if } p s t_{i} \neq j \& B_{i, j}=1\end{cases}$

where $B$ ' is the modified binary matrix representing the bays based on $p s t$ and $B ;$ c_sp stands for the count of the sub-parent. It allocates a number to each sub-parent at a given $i$-th story. $c \_s p$ has an increasing value starting from zero to $T_{-} s p_{i}$, which is the total number of sub-parent $\left(T_{-} s p\right)$ for the $i$-th story.

$T_{-} s p_{i}=1+\sum_{u=1}^{N^{u, j}-1} q ! /(q-u) ! u !$

where $T_{-} s p_{i}$ is the total number of sub-parent for the $i$-th story.

Eq. 9 gives the total number of the parent, $T_{-} p$, resulting from the combination of each sub-parent.

$T_{-} p=\prod_{i=1}^{n} T_{-} s p_{i}$

The matrix parent has three dimensions, i.e. its row, its column, and its page correspond to $n, N^{\text {tb }}$, and $p p$, respectively. The algorithm creates the matrix parent row by row on all the pages by adding in series the sub-parents $(S P)$ of the current row, as illustrated in figure 1.

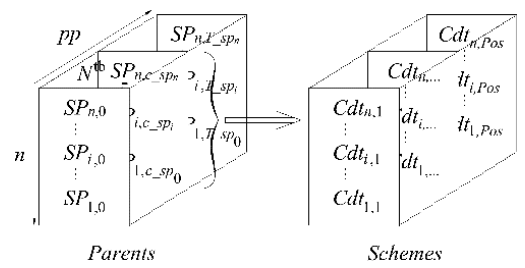

Fig. 1 Illustration of the matrices parent and scheme

\subsection{Matrix brace sections}

The schemes are the possible repartitions of a given variable related to the braces, e.g. mass, stress, or section, inside a parent. In this work, the parent provides the boundaries of the search for all possible brace sections of the structure. The authors opt for the brace section as a parameter for the creation of the schemes because the assignment of actual commercial braces for the schemes is simple with the section than the other parameters. With the 
assumption that only one discrete brace section $\left(A_{b i}^{s}\right)$ is allocated for each story, the distribution of optimal number of the brace $\left(N_{i}{ }^{\mathrm{opt}}\right)$ at the $i$-th story over the number of designable bay $\left(N_{i}^{\mathrm{db}}\right)$ gives exactly pci possible combinations for the repartition of the braces $\left(p c_{i}\right)$, or also tagged as "candidates". Eq. 10 shows the expression of $p c_{i}$.

$p c_{i}=\left(\begin{array}{c}N_{i}^{\mathrm{db}} \\ N_{i}^{\mathrm{opt}}\end{array}\right)=\frac{N_{i}^{\mathrm{db}}\left(N_{i}^{\mathrm{db}}-1\right) \ldots\left(N_{i}^{\mathrm{db}}-N_{i}^{\mathrm{opt}}+1\right)}{N_{i}^{\mathrm{opt}}\left(N_{i}^{\mathrm{opt}}-1\right) \ldots 1}$

where further details concerning $N_{i}^{\text {opt }}$ can be found in the next section.

The candidates, $C d t$, come from the manipulation of each $i$-th row of a given parent, and take the form of a vector $C d t$, constituted by element $X$. When a possible combination coincides with a designable bay, then $X_{i, j}^{k}$ takes the value of $A_{b i}^{s}$, otherwise it is zero. $k$ represents the candidate.

Eq. 11 displays the general expression of all the possible brace sections at the $i$-th story.

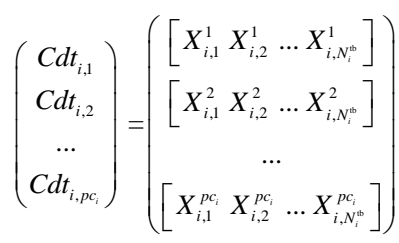

In the particular case where the final layout of the bracing systems must be symmetric, the rows of $C d t$ will be sorted out by the following condition,

$X_{i, j}^{k}=X_{i, N_{i}^{\mathrm{b}}+1-j}^{k} \quad\left(i=1, \ldots, n ; j=1, \ldots, N_{i}^{\mathrm{tb}}\right.$ and $\left.k=1, \ldots, p c_{i}\right)$

If no candidates are found, the optimal number of bay $N_{i}^{\text {opt }}$ needs a reevaluation by increasing the coefficient of reduction ci. The next section will give detailed information about $c_{i}$.

By assuming that the number of candidates found at each story starting with the first story, passing at the $i$-th story, and ending with the $n$-th story is equal to $p c_{1}, \ldots, p c_{i}, \ldots, p c_{n}$, respectively. Eq. 13 gives the total number of possible layouts of the bracing system of the structure, symbolized by Pos.

$$
\text { Pos }=p c_{1} \times \ldots \times p c_{i} \times \ldots \times p c_{n}=\prod_{i=1}^{n} p c_{i}
$$

\section{Optimal discrete brace sections derived from base-shear method}

\subsection{Overview and assumptions}

The present brace sizing optimization of seismic steel frame structure aims to reduce the total steel weight of the braces, which acts as a rough indicator of bracing construction cost. It evaluates the total optimal brace section at each story and then identifies the optimal discrete brace section related to that story. The algorithm uses a database of steel brace sizes, selected from commercially available hot-rolled, wide-flange standard steel sections, as design variables. The proposed optimization assumes that the resisting systems against to lateral loads are the braces, while the beams and columns support the gravitational load. Moreover, the deformation of the structure results from the shear and flexure displacements, which are the deformation of the braces and the rotation of the lower columns, respectively. Figure 2 and Eq. 14 come from the above assumptions.

$\delta_{i}^{\mathrm{opt}}=\delta_{\mathrm{si}}^{\mathrm{opt}}+\delta_{\mathrm{fi}}$

where $\delta_{i}{ }^{\text {opt }}$ is the optimal lateral deflection at the $i$-th story; $\delta_{s i}{ }^{\text {opt }}$ and $\delta_{f i}$ are the displacements caused by shear and flexure forces, respectively. The authors disregard the cross section of beams and columns as variables in the optimization procedures similar to the work of Moghaddam et al. [21]. However, the methodology checks the stability of all the columns under the combination of gravitational and lateral loads according to ASCE-360 [22].

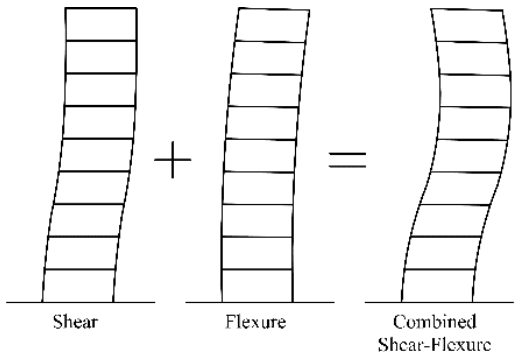

Fig. 2 Illustration of the combination shear-flexure displacement

\subsection{Flexure displacement}

Based on the work of Coeto and Teran-Gilmore [23], the bracing systems are assumed to behave globally like a beam; thus, the global flexural drifts are a consequence of the axial deformation of the support columns. Within this context, the global flexural stiffness of the structure can be estimated in the $i$-th story $\left(I_{b i}\right)$ through the full consideration of the axial areas of the support columns and the distances, which separates them.

The flexural drift, $\Delta \delta_{\mathrm{f}}$, at the $i$-th story is given by the following,

$\Delta \delta_{\mathrm{fi}}=\frac{\rho_{i}^{\text {total }}}{\sum_{j=1}^{i} h_{j}}$

where $\rho^{\text {total }}$ and $h$ are th total rotation of the slab and the structural height with respect to the ground level, respectively.

And the flexural displacement at the i-th story, as:

$\delta_{\mathrm{fi}}=\sum_{j=1}^{i} \delta_{\mathrm{f} j}$

\subsection{Optimal shear displacement}

The optimal shear drift, $\Delta \delta_{\mathrm{s}}^{\text {opt }}$, can be expressed in function of the lateral force $\left(V_{i}\right)$ and the optimal stiffness $\left(K^{\mathrm{opt}}\right)$ at the $i$-th story, as follow:

$\Delta \delta_{\mathrm{si}}^{\mathrm{opt}}=\frac{V_{i}}{K_{i}^{\mathrm{opt}}}$

Moreover, the optimal shear displacement, $\delta_{\mathrm{s}}^{\mathrm{opt}}$, at the $i$-th story, as:

$$
\delta_{\mathrm{s} i}^{\mathrm{opt}}=\sum_{j=1}^{i} \delta_{\mathrm{s} j}^{\mathrm{opt}}
$$

and

$K_{i}^{\mathrm{opt}}=\sum_{j=1}^{N_{\mathrm{ow}}^{\mathrm{op}}} \frac{E A_{b j}^{\mathrm{opt}} \cos ^{2} \alpha_{i}}{L_{i}}$

where $E$ and $L_{i}$ are respectively the braces' modulus of elasticity and the total length of the brace at the $i$-th story, $\alpha_{i}$ is the braces' inclination angle, $N_{b i}{ }^{\text {opt }}$ is the optimal number of braces at the $i$-th story, and $A_{b j}^{\text {opt }}$ is the optimal area of the brace at the $\mathrm{j}$-th bay located at the $i$-th story.

\subsection{Total optimal brace section}

The optimal number of braces at a given i-th story depends on the number of bay available to install the braces, and the type of configuration of the bracing system used. Eq. 20 describes the relation between $N_{i}^{\mathrm{opt}}$ and $A_{b j, i}^{\mathrm{opt}}$.

$A_{B i}^{\mathrm{opt}}=\sum_{j=1}^{N_{b i}^{\mathrm{ow}}} A_{b j}^{\mathrm{opt}}$ 

story.

where $A_{B i}^{\text {opt }}$ is the total sum of the optimal section of the brace at the i-th

By developing Eq. 18, and using the definition of drift, the expression of the optimal lateral displacement in function of the optimal lateral drift is giving by Eq. 21 :

$\delta_{\mathrm{s} i}^{\mathrm{opt}}=\sum_{j=1}^{i} j \Delta \delta_{\mathrm{si}-j+1}^{\mathrm{opt}}$

Substituting the expression of the optimal lateral drift in Eq. 17

$\delta_{\mathrm{si}}^{\mathrm{opt}}=\sum_{j=1}^{i} j \frac{V_{i-j+1}}{K_{i-j+1}^{\mathrm{opt}}}$

Replacing Eq. 20 into Eq.19 gives a new expression of $\Delta \delta_{\mathrm{s}}^{\mathrm{op}}$

$\Delta \delta_{\mathrm{s} i}^{\mathrm{opt}}=\frac{V_{i} L_{i}}{E A_{B i}^{\mathrm{opt}} \cos ^{2} \alpha_{i}}$

with

$L_{i}=N_{i}^{d b} l_{i} b_{T C}$

where $l_{i}$ is the effective length of the brace at the $i$-th story, and $b_{T C}$ corresponds to the number of braces forming the type of configuration used, i.e. $b_{T C}=1$ if one brace constituted the setting of interest (e.g. diagonal bracing), and $b_{T C}=2$, in the case of two braces (e.g. V-bracing).

To get the total optimal area of braces, $A_{B i}^{\mathrm{opt}}$, at the $i$-th story, Eq. 21 can be expanded as the following

$\delta_{\mathrm{s} i}^{\mathrm{opt}}=\Delta \delta_{\mathrm{s} i}^{\mathrm{opt}}+2 \Delta \delta_{\mathrm{s} i-1}^{\mathrm{opt}}+3 \Delta \delta_{\mathrm{s} i-2}^{\mathrm{opt}}+\ldots+(i-1) \Delta \delta_{\mathrm{s} 2}^{\mathrm{opt}}+i \Delta \delta_{\mathrm{s} 1}^{\mathrm{opt}}$

Subtract both sides of Eq. 25 with $\delta_{s i-1}^{\text {opt }}$, then use Eq. 14 to get the following

$\left(\left(\delta_{i}^{\mathrm{opt}}-\delta_{i-1}^{\mathrm{opt}}\right)-\left(\delta_{\mathrm{f} i}-\delta_{\mathrm{f} i-1}\right)\right)=\Delta \delta_{\mathrm{s} i}^{\mathrm{opt}}+\sum_{j=1}^{i-1} \Delta \delta_{\mathrm{s} j}^{\mathrm{opt}}$

The optimal structural drift, $\Delta \delta_{i}{ }^{\text {opt }}$, and the flexural drift, $\Delta \delta_{f i}$, at the $i$-th story can be identified in Eq. 26, Therefore, the sum of $\Delta \delta_{\mathrm{s}}^{\mathrm{opt}}$ below the i-th level can be represented by $\Delta \delta_{\mathrm{s}}^{\prime}$ as shown in Eq. 27 .

$\Delta \delta_{\mathrm{s} i}^{\prime}=\sum_{j=1}^{i-1} \frac{V_{j} L_{j}}{E A_{B j}^{\mathrm{opt}} \cos ^{2} \alpha_{j}} \quad$ with $\Delta \delta_{\mathrm{s} 1}^{\prime}=0$

$\Delta \delta^{\text {opt }}$ of the $i$-th story depends on the target drift, $\Delta \delta^{\text {target }}$, of the selected structural performance level, and it bounds by the following condition.

$$
\Delta \delta_{i}^{\mathrm{opt}} \leq \Delta \delta^{\mathrm{target}}
$$

A proposed way to estimate $\Delta \delta^{\text {opt }}$ is to multiply $\Delta \delta^{\text {target }}$ by an index ratio corresponding to the effect of the external force inputted into the structure. The authors use the overturning moment distribution to calculate this index ratio, $\mu$, at the $i$-th story because of its relation to the height of the structure. Eq. 29 comes from the assumption that the n-th story displays the highest drift.

$\Delta \delta_{i}^{\mathrm{opt}}=\mu_{i} \Delta \delta^{\mathrm{target}}$

Eq. 30 gives the final expression of $A_{B i}^{\mathrm{opt}}$.

$A_{B i}^{\mathrm{opt}}=\frac{V_{i} L_{i}}{\left(\mu_{i} \Delta \delta^{\mathrm{target}}-\Delta \delta_{\mathrm{fi}}-\Delta \delta_{\mathrm{si}}^{\prime}\right) E \cos ^{2} \alpha_{i}}$

\subsection{Optimal discrete brace section}

The optimal discrete brace section, $A_{b i}^{\mathrm{s}}$, selected from the predefined database, comes from the identification of the optimal brace section, $A_{b i}^{\mathrm{opt}}$. This database contains $\mathrm{m}$ sections that are sorted from the smallest to the biggest section, which refer to $A_{b}^{\mathrm{s} 1}$ and $A_{b}^{\mathrm{sm}}$, respectively. The following condition bounds the value of $A_{b i}^{\text {opt }}$ :

$A_{b}^{\mathrm{s} 1} \leq A_{b i}^{\mathrm{opt}} \leq A_{b}^{\mathrm{sm}}$

The sizing optimization selects a single discrete section for all the braces at the $i$-th story because of the following reasons. 1) Multiple sections of brace suggest the presence of weak brace, i.e. the dissipation of the lateral force at the $i$-th story by the braces will not be uniform. 2) This weak brace might lead to earlier damage in the frame of the structure, because, as the braces are located at the same story, the weak brace reaches its strength limit earlier that the other ones. 3) Moreover, in many design projects, the one brace section choice at each i-th story is the way to design the bracing system.

Therefore, $A_{b i}^{\text {opt }} \lim$ can be calculated by using Eq. 20,

$A_{b i}^{\mathrm{opt}}=\frac{A_{B i}^{\mathrm{opt}}}{N_{b i}^{\mathrm{opt}}}$

The optimal number of brace at the $i$-th story is given by the following,

$N_{b i}^{\mathrm{opt}}=b_{T C}\left(N_{i}^{\mathrm{db}}-c_{i}\right)$

where $c_{i}$ is a coefficient of reduction that is initially equal to zero. The augmentation of its value from zero to $\left(N_{i}^{\mathrm{db}}-1\right)$ permits to identify all the candidates at the $i$-th story, as expressed in Eq. 10. $A_{b i}^{\mathrm{s}}$ corresponding to $A_{b i}^{\text {opt }}$ is the section $A_{b}^{\text {sdata }}$ that has the smallest difference between the discrete and the exact sections. Eq. 34 expresses that statement.

$$
g\left(A_{b i}^{\mathrm{s}} \mid A_{b}^{\text {sdata }}\right)=\min \left(A_{b}^{\text {sdata }}-A_{b i}^{\mathrm{opt}}\right) \quad(\text { data }=m, m-1, \ldots, 1)
$$

s.t.: $\quad A_{b i}^{\mathrm{s}} \leq A_{b i-1}^{\mathrm{s}}$

where the selected discrete section at the $i$-th story is the maximum allowable section to be chosen at the $i+1$ th story. This restriction aims to avoid any formation of soft stories in the building. When the algorithm finds no discrete section, it assigns null sections for that candidate, and then increases $c_{i}$ to get a new optimal number of the brace, $N_{i}^{\text {opt }}$.

The sizing optimization minimizes the total volume of braces, in which the upper bound value $A_{b}^{s m}$ constrains the discrete section $A_{b i}^{s}$.

$\min : \quad v_{z}^{\text {scheme }}=\sum_{i=1}^{n} L_{i} A_{b i}^{s}$

s.t.: $\quad 0<A_{b i}^{s} \leq A_{b}^{s m}$

where $v_{z}^{\text {scheme }}$ is the optimal volume of the braces for the $z$-th scheme.

Some parents might give the same scheme. In that case, the algorithm saves the information of the first scheme and ignores the others. The next step of the proposed methodology will utilize these series of different schemes to identify the optimal one.

\section{Optimal design of bracing systems using collapse safety assessment}

\subsection{Overview}

The present design optimization of braced steel structures can be conceptually stated as:

Objective: Maximize the $C M R$ of the structure, which is a safety indicator; Variable: The brace location and section, mathematically expressed by the matrix $S P$;

Constraint: Candidates are non-null vectors, i.e. each level of the steel frame structure should at least have one brace. 


\subsection{Collapse safety evaluation}

FEMA [11] discusses the concept of CMR in full details and defines its value as follows

$$
C M R_{S c h}=\frac{I M_{50 \%}}{I M_{M C E}}
$$

where $I M_{50 \%}$ corresponds to the intensity measure when $50 \%$ of the ground motion causes structural collapse and $I M_{M C E}$ is the intensity measure of the maximum considered earthquake causing structural collapse.

The basic expression of the cumulative distribution function that defines the collapse fragility curve is as follow [24-26]:

$$
P(C \mid I M)=\Phi\left(\frac{\ln \left(I M \theta^{-1}\right)}{\beta}\right)
$$

where $P(C \mid I M)$ is the probability that a ground motion with $I M$ causes the structure to collapse; $\Phi()$ is the standard normal cumulative distribution function; $\theta$ is the median of the fragility function, and $\beta$ is the dispersion of $I M$.

Eq. 37 can be rewritten as:

$$
P(C \mid I M)=\frac{1}{2}\left[1+\operatorname{erf}\left(\frac{\ln \left(I M \theta^{-1}\right)}{\sqrt{2} \beta}\right)\right]
$$

Then $I M$ is isolated as follow:

$$
I M=e^{\sqrt{2} \beta \operatorname{Rootof}(-\operatorname{erf}(-Z)+2 P(C \mid M)-1)} \theta
$$

By substituting each $I M$ in Eq. 36, and incorporating Eq. 38 with $I M$ equal to $I M_{M C E}$ gives a simple expression of $C M R$ :

$$
C M R_{S c h}=\frac{\theta}{I M_{M C E}}
$$

More frequently, the spectral acceleration for a specified period and damping quantify the parameter $I M$. Thus, by using the expression of the spectral acceleration for the given period $T_{1}$, and replacing the expression of the median of the fragility curve $\theta$ by the one used in the empirical equations, the following is obtained:

$$
C M R_{S c h}= \begin{cases}\frac{A_{y}}{S_{D S}}\left(R_{c}\right)_{\zeta s s} & \text { if } T_{0} \leq T_{1} \leq T_{S} \\ \frac{A_{y} T_{1}}{S_{D 1}}\left(R_{c}\right)_{\zeta_{s s}} & \text { if } T_{S}<T_{1} \leq T_{L}\end{cases}
$$

where $A_{y}$ is the pseudo-acceleration where the system yields, which is given by the force-deformation relation from the modal pushover analysis (MPA); $\left(R_{c}\right)_{\zeta}$ is the collapse strength ratio with damping $\zeta$ and has an empirical equation in the work of Han, et al. [9]. $S_{D S}$ and $S_{D 1}$ are the design spectrum response acceleration parameters at short and 1-s periods, respectively. The transition periods $T_{0}, T_{S}$ and $T_{L}$ are well detailed in ASCE-7 [27].

$$
C M R_{S c h}= \begin{cases}\frac{A_{y}}{S_{D S}}\left[1+\mu_{c}\left(1-e^{-2.5 T_{1}}\right)\left(-0.38 \mu_{c}^{a} \alpha_{c}^{-1}\right)^{\mu_{c}^{-b}+0.03 T_{i}-0.03 \ln T_{i}}\right] & \text { if } T_{0} \leq T_{1} \leq T_{S} \\ \frac{A_{y} T_{1}}{S_{D 1}}\left[1+\mu_{c}\left(1-e^{-2.5 T_{1}}\right)\left(-0.38 \mu_{c}^{a} \alpha_{c}^{-1}\right)^{\mu_{c}^{-b}+0.03 T_{1}-0.03 \ln T_{i}}\right] & \text { if } T_{S}<T_{1} \leq T_{L}\end{cases}
$$

If $\zeta$ is other than five percent, the following expressions are used

$\left(R_{c}\right)_{\zeta}=C_{\zeta}\left(R_{c}\right)_{\zeta_{s s}}$

with

$C_{\zeta}=\left\{1-\left[\frac{(0.07 \ln \zeta+0.20)}{T_{1}^{0.38}\left(-\alpha_{c}\right)^{-0.26} \mu_{c}^{-0.44}}\right]\right\}$

where the controlling parameters for the strength-limited bilinear model [28], illustrated in figure 3, are initial stiffness $k_{e}$, second slope stiffness $k_{s}=\alpha_{s} k_{\mathrm{e}}$, negative slope stiffness $k_{c}=\alpha_{c} k_{e}$, yield strength $f_{y}$, yield displacement $u_{y}$, maximum strength $f_{c}$, and displacement $u_{c}$ at $f_{c}$, ductility coefficient $\mu_{c}=u_{c} / u_{y}$ and residual strength $f_{l}=\lambda f_{y}$. Related to those parameters are $T_{1}$, the small amplitude natural vibration period associated with initial stiffness $k_{e}$; and $A_{y}$, the pseudoacceleration related to yield strength $f_{y}$.

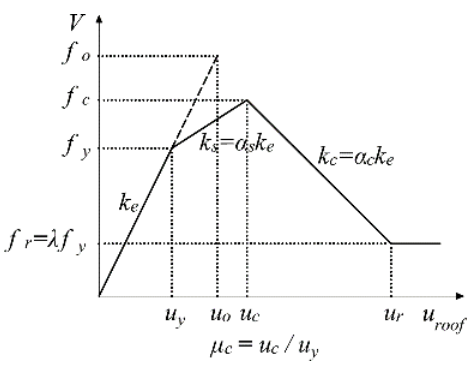

Fig. 3 Idealized pushover curve

The authors idealized the pushover curves by using the procedure described in the work of Han, et al. [9]. The lateral loads use in the MPA followed the procedure established by Chopra and Goel [8]. Also, the limitations concerning the empirical equations, Eq. 42, Eq. 43, and Eq. 44 depend on the used design spectrum response acceleration parameters, $S_{D S}$ and $S_{D 1}$ and the boundaries of the $C M R$. Deduced from Eq. 36, this minimum $C M R$ value is not inferior nor equal to one. On the other hand, its maximum value is difficult to designate with an exact certainty because any changes in the parameters of the $C M R$ calculation might give a value bigger than the supposed highest one. This latter confirms the conclusion reached by Xian, et al. [14] that $C M R$ is a case-dependent factor. Therefore, the issue of an acceptable maximum $C M R$ needs a deeper investigation, which is out of the scope of the current work. For the sake of simplicity and understanding the relationship between the CMR and all the parameters in Eq. 43, the authors identify and use one reasonable maximum $C M R$ throughout the current and remaining sections of this work. By reviewing some $C M R$ values in the literature [12, 29-31], the authors observe that $I M_{50 \%}$ equal to three times of $I M_{M C E}$ covers most of the cases, which leads to a $C M R$ value of three. Moreover, a structural collapse resistance of three times the maximum considered earthquake in the design is satisfactory.

To determine the range of the parameters the above empirical equations according to the boundaries of the $C M R(1<C M R \leq 3)$, the authors utilized the range in the work of Han, et al. [9] (see table 1).

Figure 4 illustrates the variation of the $C M R$ areas with different damping values. By establishing an upper bound of the $C M R$, the maximum range of $T_{1}$ follows the expression of the line formed by the considered damping. Examples of this expression lay in figures 4-6, and show the influence of the extreme values of $\alpha \mathrm{s}$ and $\alpha \mathrm{c}$ on the area of the $C M R$. Figure 7 uses all the parameters to create several $C M R$ curves depending on different damping values. The missing data in figure $7 \mathrm{~b}$ are above the upper limit of the $C M R$, which signifies that the range of $\mu_{c}$ might decrease depending on the damping.

Table 1

Variables considered for the braced steel structures

\begin{tabular}{cc}
\hline Parameters & Range \\
\hline$T_{1}$ & $0.2-4.0$ \\
$\alpha_{s}$ & $0.00-0.20$ \\
$\alpha_{c}$ & $-0.1--0.5$ \\
$\mu_{c}$ & $1-6$ \\
$\zeta$ & $2-20$ \\
\hline
\end{tabular}




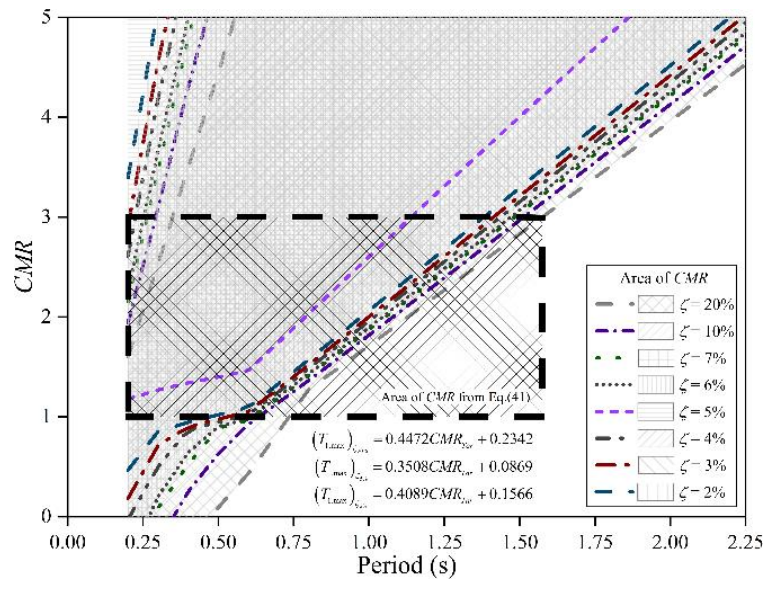

Fig. 4 Area of $C M R$ with different $\zeta$

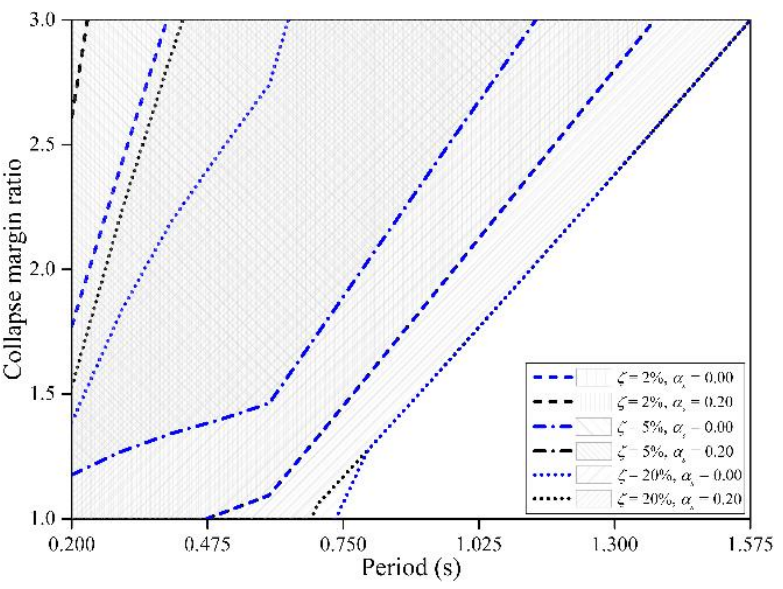

Fig. 5 Area of $C M R$ with the parameters $\zeta$, and $\alpha_{s}$

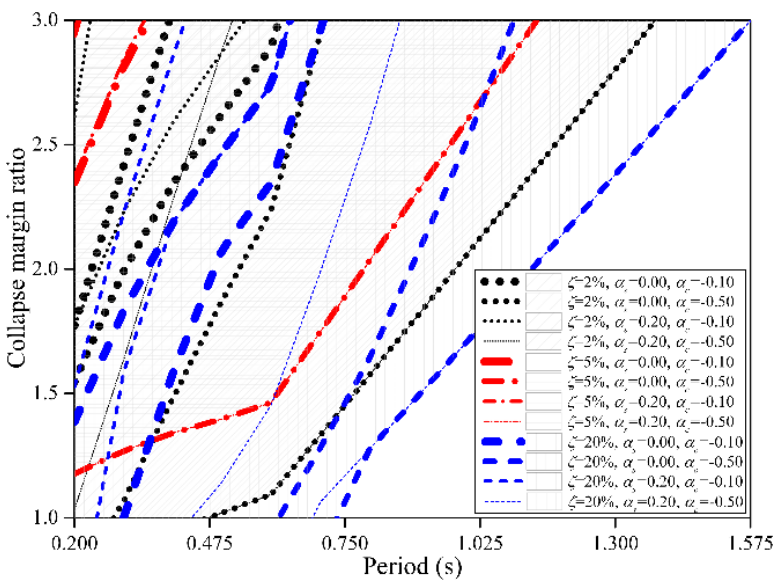

Fig. 6 Area of $C M R$ with the parameters $\zeta, \alpha_{s}$, and $\alpha_{c}$

4.3. Optimal brace scheme

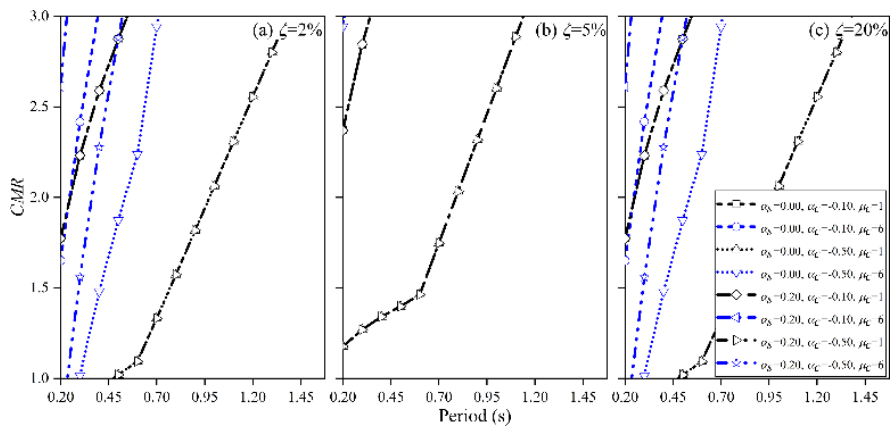

Fig. $7 C M R$ curves for different damping values (a) $\zeta=2 \%$; (b) $\zeta=5 \%$; (c) $\zeta=20 \%$
The last step of the proposed methodology analyses the schemes from the parents and identifies the safest one among them, i.e. the scheme that has the highest $C M R$. The proposed methodology utilizes the aforementioned upper bound of the $C M R$ and assumes that the optimal safety criteria is related to that value. This safety value will be the target of the search of the safest scheme by the utilization of the safety index $(S I)$ in Eq. 45

$S I=\frac{C M R_{\text {scheme }}}{C M R_{\text {target }}}$

where $C M R_{\text {scheme }}$ and $C M R_{\text {target }}$ are the $C M R$ of the investigated scheme and the target $C M R$ for the structural design, respectively.

In this proposed methodology, the search for the optimal scheme has two possible stopping criteria: 1) when one of the schemes presents a SI superior or equal to one, and 2) all the possible schemes are analyzed. Therefore, the scheme that has the largest $S I$ is the safest one. Figure 8 shows the overall steps for the proposed optimization.

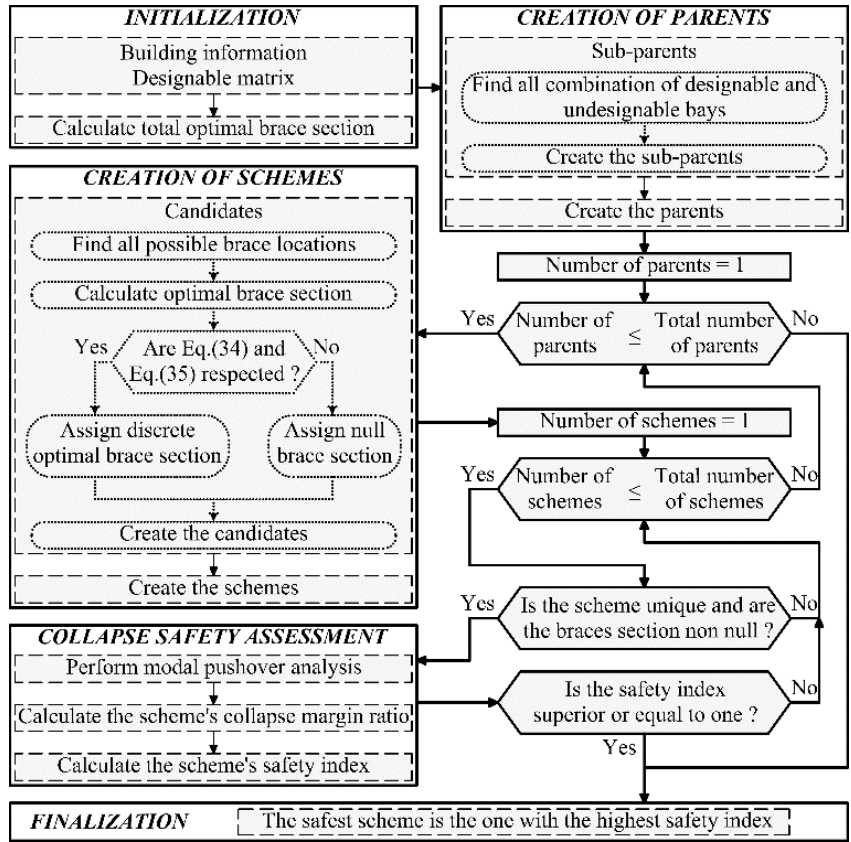

Fig. 8 Flowchart of the proposed design optimization

\section{Numerical examples}

\subsection{Modeling using OpenSees}

The authors choose OpenSees because of its popularity and its rapid and efficient analytic simulation $[32,33]$. They follow the "line-element" approach [34] to model the CBFs. Each investigated model considers the gravity load during the simulation process. The seismic design of the CBFs follows the requirements described in ASCE-7 [27] concerning the special concentrically braced frames with response modification coefficient, $R=6$, overstrength factor, $\Omega_{0}=2$, and deflection amplification factor, $C_{d}=5$. As the behavior of the bracing system affects the entire structure, their modeling has to be precise. Ten nonlinear fiber elements form the braces, as suggested by Uriz and Mahin [35] to fulfill the minimum number of elements for global and local analyses of the bracing systems. The latter adopt a quadratic out-of-plane imperfection equal to their effective length divided by one thousand and a corotational geometric transformation. The gusset plate connection uses the modeling method proposed by Hsiao et al. [36], which have a rotational hinge at both ends of the brace to simulate their behavior. The "zeroLength" element represents the rotational hinge by using the command "uniaxialMaterial" with the material behavior "Steel 02", which depicts the fatigue of the material [37]. Figure 9 gives globa and close-up views of the modeling method for the braced frame in OpenSees. 


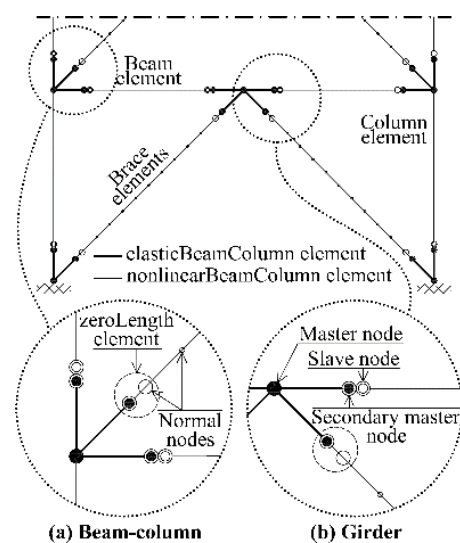

Fig. 9 OpenSees modeling method

\subsection{Building models}

The authors investigate four steel frame structures divided into two cases, as shown in figure 10. The cases A present some undesignable bays on their sides, while the cases B have none. In both cases, the number of stories differentiates the models. The dead and live loads are $6500 \mathrm{~N} / \mathrm{mm}^{2}$ and 2000 $\mathrm{N} / \mathrm{mm}^{2}$, respectively. Chevron with a hallow shape section is the type of CBFs configuration considered for the analyses. All cases use $C M R_{\text {target }}=3$ and $\zeta=5 \%$. For the computational analyses, the authors used one workstation equipped with thirty-two core processor of $2.6 \mathrm{GHz}$, and sixty-four gigabits of memory size.
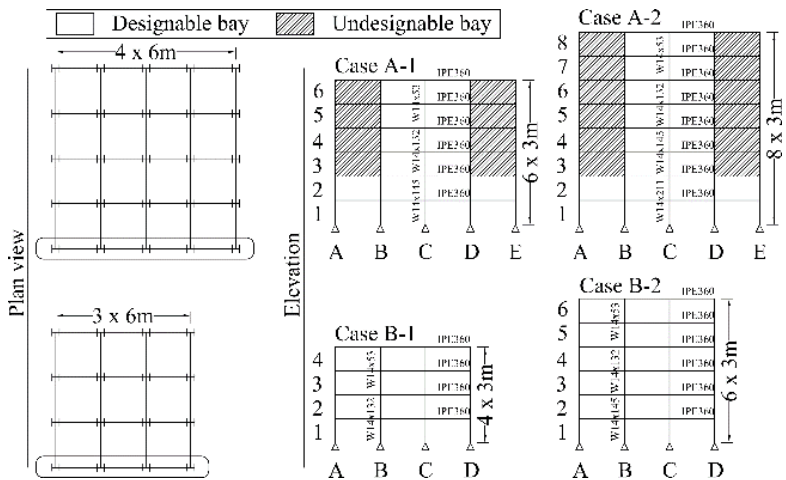

Fig. 10 Plan view and elevation of the buildings

\subsection{Results and discussions}

Figures 11-12 present the outcomes of the cases A and B. The presentation of the scheme samples illustrates the manipulation of the bracing systems done by the probabilistic analysis using multi-element removal and the sizing optimization. The two vertical axis represent the CMR and the SI of the schemes. The points depict the CMR value of each individual scheme, while the line steps indicate the maximum value of SI. The table at the bottom of the figures 11-12 are the sample representation of the evolution of the schemes throughout the analysis, and the safest scheme for each cases is displayed at the bottom right inside.

Two criteria define the safest schemes: 1) the safety index reaches its maximum value ( $S I=1)$. This first criterion applies to the case A2 and B2. 2) The number of possible scheme is reached, thus the scheme with the highest SI is the one. This second criterion appears with the case A1 and B1. Case A2 and B2 reached their maximum safety index with the fifty-ninth and the four hundred and thirty-eighth schemes, respectively, while the seventy-fourth and the hundred and nineteen-ninth schemes of the case A1 and B1 were the safest among their respective groups. In all four cases, the schemes with symmetrical layout have high $S I$ value.

The authors observed that, depending on the size of the matrix $B$, the search of the possible schemes might become tedious when the $C M R_{\text {target }}$ is overestimated, or it is not included in the optimization. For instance, without $C M R_{\text {target }}$, the case B1 took twenty-nine hours to complete (55 parents and 181 schemes), while the case B2 lasted for nineteen days (234 parents and 1944 schemes). Despite this lengthy analysis, the proposed methodology assesses the collapse margin of an approximately hundred structures in one day, which is clearly more practical and very quick compared to the earlier works in this area. $C M R_{\text {target }}$ within the range of four to five seems to give a reasonable convergence

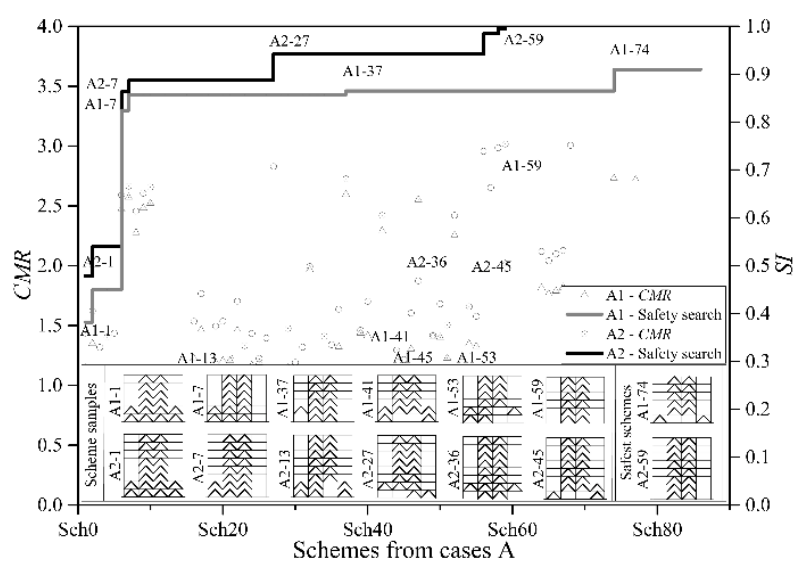

Fig. 11 Result of the design optimization of the cases A

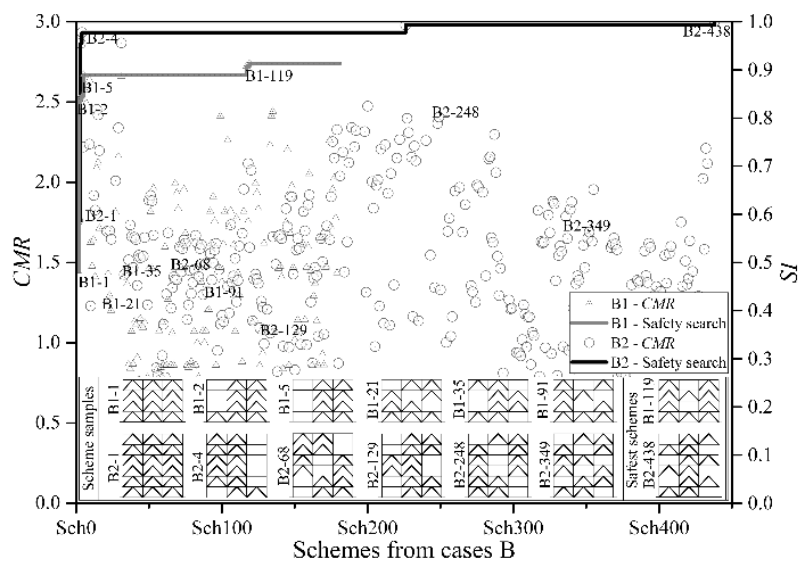

Fig. 12 Result of the design optimization of the cases B

of the safety search. Although it allows shortening the analysis time, the proposed method might leave behind some realistic schemes when the selection of the schemes implements with general conditions. In this work, the scheme's selection followed the status of uniqueness and non-null brace sections. More realistic results should appear earlier in the analysis if the condition of symmetric schemes was considered. Eq. 12 gives the implementation of this last condition in the creation of the schemes. The cases A are clear examples of the above statement. If symmetricity was considered in the optimization algorithm, their safest schemes would appear sooner during the analysis; also, it will reduce the number of selected schemes, which reduces the computational time as well. Therefore, for research purpose, to show the full extent of the proposed methodology, the authors opt to analyze both symmetric and asymmetric schemes.

Finding an optimal brace layout based on safety criteria is a complicated problem that needs to be studied on a case-by-case basis. The utilization of probabilistic analysis using multi-element removal and a combination of MPA and the empirical equation of $C M R$ allow the proposed method to achieve that goal. The proposed sizing optimization minimizes the section of the brace systems by selecting the optimal discrete section based on the optimal number of brace and the total optimal brace section. To obtain a more accurate brace section design, all columns and beams sizes should be accounted for in the optimization.

\section{Conclusions}

A new methodology incorporating optimization technique with probabilistic analysis using multi-element removal has been introduced to improve the safety design of steel structure with concentric braces. It has proved to be a potentially useful tool for the understanding of the optimal placement for the bracing systems. The proposed methodology is capable of a quick and practical estimation of the collapse margin of several structures in a short time compared to the prior methods in this field. It considered the braces location and section as variables and optimized the structure according to the safety index of the selected schemes.

The range of the period $T_{1}$ relates closely to the target $C M R$ and the damping. A higher upper bound value of the $C M R$ is possible, but also, it 
modifies the range of some parameters such as $T_{1}$ and $\mu_{c}$.

To efficiently use the proposed methodology, the authors suggest at least the following conditions for the selection of the schemes: uniqueness, non-null brace sections, and symmetricity. Further conditions such as specific brace cross-sections (personalized database), and predetermined brace layout patterns (case A has an inverted T pattern) can be included in the creation and selection of the scheme to channel the safety search toward particular schemes. Moreover, the results from the numerical examples highlight that symmetrical brace layouts are safer than the asymmetrical ones, and fully braced structures do not have high safety index.

The findings above are based on the assumptions and limitations adopted in this study, which are the focus on the steel frame structures controlled by its fundamental mode, the braces parameters (location, number, and cross-section), the unchanged main frame, and the constraint regarding the range of the parameters of the empirical equation. The proposed methodology provides a useful basis for more comprehensive seismic collapse assessment of steel structures if the design of the braces and the structural frame are simultaneously considered and more general parameters about the CMR calculations are developed.

\section{Acknowledgments}

The research reported in this paper was financially supported by the National Natural Science Foundation of China (Grant No. 51878123).

\section{References}

[1] Stromberg L.L., Beghini A., Baker W.F. and Paulino G.H., "Topology optimization for braced frames: Combining continuum and beam/column elements", Engineering Structures, 37, April, 106-124, 2012

[2] Vamvatsikos D. and Cornell C.A., "Incremental dynamic analysis", Earthquake Engineering \& Structural Dynamics, 31(3), 491-514, 2002.

[3] Han S.W. and Chopra A.K., "Approximate incremental dynamic analysis using the modal pushover analysis procedure", Earthquake Engineering and Structural Dynamics, 35(15), $1853-1873,2006$

[4] Hamidia M., Filiatrault A. and Aref A., "Simplified seismic sidesway collapse analysis of frame buildings", Earthquake Engineering \& Structural Dynamics, 43(3), 429-448, 2014.

[5] Hardyniec A. and Charney F., "A new efficient method for determining the collapse margin ratio using parallel computing", Computers and Structures, 148, February, 14-25, 2015.

[6] Liu S.W., Bai R. and Chan S.L., Dynamic Time-history Elastic Analysis of Steel Frames Using One Element per Member, presented at the Structures, 2016

[7] Liu S.W., Liu Y.P. and Chan S.L., "Pushover analysis by one element per member for performance-based seismic design", International Journal of Structural Stability and Dynamics, 10(01), 111-126, 2010

[8] Chopra A.K. and Goel R.K., "A modal pushover analysis procedure for estimating seismic demands for buildings", Earthquake Engineering \& Structural Dynamics, 31(3), 561-582, 2002.

[9] Han S.W., Moon K.H. and Chopra A.K., "Application of MPA to estimate probability of collapse of structures", Earthquake Engineering and Structural Dynamics, 39(11), 1259-1278, 2010

[10] Moon K.H., Han S.W., Lee T.S. and Seok S.W., "Approximate MPA-based method for performing incremental dynamic analysis", Nonlinear Dynamic, 67(4), 2865-2888, 2012.

[11] FEMA, Quantification of Building Seismic Performance Factors, Federal Emergency Management Agency, Washington, DC, 2009.

[12] Ou X., He Z. and Ou J., "Parametric study on collapse margin ratio of structure", Journal of Central South University, 21(6), 2477-2486, 2014

[13] Farsangi E.N., Yang T.Y. and Tasnimi A.A., "Influence of concurrent horizontal and vertical ground excitations on the collapse margins of non-ductiles RC frame buildings", Structural Engineering and Mechanics, 59(4), 653-669, 2016.
[14] Xian L., He Z. and Ou X., "Incorporation of collapse safety margin into direct earthquake loss estimate", Earthquakes and Structures, 10(2), 429-450, 2016.

[15] Tirca L., Chen L. and Tremblay R., "Assessing collapse safety of CBF buildings subjected to crustal and subduction earthquakes", Journal of Constructional Steel Research, 115, December, 47-61, 2015.

[16] Kicinger R., Arciszewski T. and De Jong K., "Evolutionary computation and structural design: A survey of the state-of-the-art", Computers and Structures, 83(23-24), 1943-1978, 2005.

[17] Pezeshk S. and Camp C.V., State of the Art on the Use of Genetic Algorithms in Design of Steel Structures, American Society of Civil Engineers, Reston, VA, 2002.

[18] Schafer B.W. and Bajpai P., "Stability degradation and redundancy in damaged structures", Engineering Structures, 27(11), 1642-1651, 2005.

[19] Gholizadeh S. and Poorhoseini H., "Seismic layout optimization of steel braced frames by an improved dolphin echolocation algorithm", Structural and Multidisciplinary Optimization, 54(4), October 01, 1011-1029, 2016.

[20] Liu M., "Progressive collapse design of seismic steel frames using structural optimization", Journal of Constructional Steel Research, 67(3), 322-332, 2011.

[21] Moghaddam H., Hajirasouliha I. and Doostan A., "Optimum seismic design of concentrically braced steel frames: concepts and design procedures", Journal of Constructional Steel Research, 61(2), 151-166, 2005.

[22] ASCE-360, "Specification for Structural Steel Buildings," American Society of Civil Engineers, Reston, VA, 2010.

[23] Coeto G. and Teran-Gilmore A., Stiffness-Based Sizing of Bracing Systems for Tall and Slender Buildings, Proceeding of the $15^{\text {th }}$ World Conference on Earthquake Engineering, Lisbon, Portugal, 10, 2012.

[24] Baker J.W., "Efficient analytical fragility function fitting using dynamic structural analysis", Earthquake Spectra, 31(1), 579-599, 2015

[25] Bradley B.A. and Dhakal R.P., "Error estimation of closed-form solution for annual rate of structural collapse", Earthquake Engineering and Structural Dynamics, 37(15), 1721-1737, 2008.

[26] Porter K., Kennedy R. and Bachman R., "Creating fragility functions for performance-based earthquake engineering", Earthquake Spectra, 23(2), 471-489, 2007

[27] ASCE-7, Minimum Design Loads for Buildings and Others Structures, American Society of Civil Engineers, Reston, VA, 2010.

[28] Ibarra L.F., Medina R.A. and Krawinkler H., "Hysteric models that incorporate strength and stiffness deterioration", Earthquake Engineering \& Structural Dynamics, 34(12), 1489-1511, 2005.

[29] Nobahar E., Farahi M. and Mofid M., "Quantification of seismic performance factors of the buildings consisting of disposable knee bracing frames", Journal of Constructional Steel Research, 124, September, 132-141, 2016.

[30] Shin D.H. and Kim H.J., "Influential properties of hysteretic energy dissipating devices on collapse capacities of frames", Journal of Constructional Steel Research, 123, August, 93 $105,2016$.

[31] Hamidia M., Filiatrault A. and Aref A., "Seismic collapse capacity-based evaluation an design of frame buildings with viscous dampers using pushover analysis", Journal of Structural Engineering, 141(6), 04014153, 2015.

[32] Elhami Khorasani N., Garlock M.E.M. and Quiel S.E., "Modeling steel structures in OpenSees: Enhancements for fire and multi-hazard probabilistic analyses", Computers and Structures, 157, September, 218-231, 2015.

[33] Lu X., Xie L., Guan H., Huang Y. and Lu X., "A shear wall element for nonlinear seismic analysis of super-tall buildings using OpenSees", Finite Elements in Analysis and Design, $98,14-25,2015$

[34] Terzic V., Modeling SCB Frames Using Beam-Column Elements, Berkeley, CA, 2013.

[35] Uriz P. and Mahin S.A., Toward Earthquake-Resistance Design of Concentrically Braced Steel-Frame Structures, Pacific Earthquake Engineering Research Center, Berkeley, CA, 2008 .

[36] Hsiao P.C., Lehman D.E. and Roeder C.W., "Improved analytical model for special concentrically braced frames", Journal of Constructional Steel Research, 73, June, 80-94, 2012

[37] Mazzoni S., McKenna F., Scott M.H. and Fenves G.L., Open System for Earthquake Engineering Simulation (OpenSees): Command Language Manual, Available at http://opensees.berkeley.edu/OpenSees/manuals/usermanual/, 2007. 